\title{
Expression of TIGIT/CD155 and correlations with clinical pathological features in human hepatocellular carcinoma
}

\author{
XIANGGUO DUAN $^{1,2^{*}}$, JUANXI LIU $^{1 *}$, JIANJIAN CUI $^{3 *}$, BIN MA $^{4}$, QIUNAN ZHOU ${ }^{1}$, \\ XIAOJUAN YANG ${ }^{1}$, ZHENHUI LU ${ }^{2}$, YONG DU ${ }^{2}$ and CHUNXIA $\mathrm{SU}^{5}$ \\ ${ }^{1}$ Department of Laboratory Medicine, College of Clinical Medicine, Ningxia Medical University; \\ ${ }^{2}$ Department of Laboratory Surgery, General Hospital of Ningxia Medical University; \\ ${ }^{3}$ Department of Clinical Laboratory, Ningxia Chinese Medicine Research Center; \\ ${ }^{4}$ Department of Oncology Surgery, The First People's Hospital of Yinchuan; \\ ${ }^{5}$ Department of Pathogen Biology and Immunology, School of Basic Medical Science, \\ Ningxia Medical University, Yinchuan, Ningxia Hui Autonomous Region 750004, P.R. China
}

Received January 3, 2019; Accepted June 25, 2019

DOI: $10.3892 / \mathrm{mmr} .2019 .10641$

\begin{abstract}
T cell immunoglobulin and ITIM domain (TIGIT) is a recently identified $\mathrm{T}$ cell coinhibitory receptor. Studies have shown that TIGIT is expressed in colon adenocarcinoma, uterine corpus endometrioid carcinoma, breast carcinoma and kidney renal clear cell carcinoma. However, the role of the TIGIT/human poliovirus receptor (CD155) pathway in the pathogenesis of hepatocellular carcinoma (HCC) remains to be elucidated. In the present study, the expression of TIGIT and CD155 in HCC tissues and peripheral blood were determined, and correlations among TIGIT, CD155, TIGIT $^{+} \mathrm{CD}^{+} \mathrm{T}$ cells, TIGIT $^{+}$regulatory $\mathrm{T}$ (Treg) cells and $\alpha$-fetoprotein (AFP) were investigated in order to identify a potential target for diagnosing and treating HCC. Immunohistochemistry, reverse transcription-quantitative PCR analysis and western blotting were used to examine the expression of TIGIT and CD155 in cancerous tissues and peripheral blood collected from patients with HCC. The frequency of $\mathrm{TIGIT}^{+} \mathrm{CD}^{+}{ }^{+} \mathrm{T}$ cells and $\mathrm{TIGIT}^{+}$ Treg cells and the concentration of inflammatory cytokines secreted by $\mathrm{T}$ cell subsets were analyzed by flow cytometry and a Merck Milliplex assay. Correlations between the frequency of TIGIT $^{+} \mathrm{CD}^{+}{ }^{+} \mathrm{T}$ and TIGIT $^{+}$Treg cells and AFP were analyzed using Spearman's rank correlation test. With the degree of cancerous differentiation from high to low, the
\end{abstract}

Correspondence to: Ms. Chunxia Su, Department of Pathogen Biology and Immunology, School of Basic Medical Science, Ningxia Medical University, 1160 Shengli Street, Yinchuan, Ningxia Hui Autonomous Region 750004, P.R. China

E-mail: 1651085195@qq.com

*Contributed equally

Key words: hepatocellular carcinoma, $\mathrm{T}$ cell immunoglobulin and ITIM domain, CD155, $\alpha$-fetoprotein, pathological features expression levels of TIGIT and CD155 were upregulated in the cancerous tissues from patients with $\mathrm{HCC}$. TIGIT $^{+} \mathrm{CD}^{+}{ }^{+} \mathrm{T}$ cell and TIGIT $^{+}$Treg cell frequencies were decreased in peripheral blood from postoperative patients with HCC. The increased expression of TIGIT was positively correlated with the level of AFP. These results indicate that co-inhibitory receptor TIGIT may be involved in the pathogenesis of HCC and represent a novel target for the diagnosis and treatment of HCC.

\section{Introduction}

Hepatocellular carcinoma (HCC) is a common malignant tumor that is harmful to human health. HCC ranks fifth highest in incidence and second in cancer-related mortality worldwide, with $>50 \%$ of HCC cases occurring in China $(1,2)$. Approximately $75 \%$ of $\mathrm{HCC}$ cases are caused by chronic infection with hepatitis $B$ virus or hepatitis $C$ virus $(3,4)$. There are almost 1,400,000 cases of chronic liver disease-associated mortality annually (5), and HCC is the predominant cause of mortality in patients with cirrhosis (6). When diagnosed early, the prognosis of HCC is good, with a 5-year survival rate of $>70 \%$; however, the prognosis of $\mathrm{HCC}$ is poor when diagnosed late, with a 5-year survival rate of $<16 \%$ (5). As $\mathrm{HCC}$ is highly resistant to chemotherapy, immune therapy is an attractive treatment option, as the inflammatory tumor microenvironment is associated with improved survival $(7,8)$. Previous studies have shown that the aggregation of activated regulatory $\mathrm{T}$ (Treg) cells in the tumor microenvironment is one of the inhibitory mechanisms leading to immune evasion of HCC (9-11). However, inhibitory receptors on immune cells may contribute to the suppression of antitumor immunity in the tumor microenvironment.

A novel inhibitory receptor, $\mathrm{T}$ cell immunoglobulin and ITIM domain (TIGIT), has been shown to be expressed on active T cells, natural killer cells (NK cells) (7) and tumor cells, including gastric cancer cells (8). Human poliovirus receptor, CD155, is the physical ligand of TIGIT. TIGIT/CD155 engagement can inhibit $\mathrm{T}$ cell responses in a cell-intrinsic manner by 
directly targeting the $\mathrm{T}$ cell receptor (TCR) signaling cascade and inducing tolerogenic dendritic cells (DCs) (9-11). Treg cells selectively inhibit proinflammatory $\mathrm{T}$ helper 1 (Th1) and $\mathrm{T}$ helper 17 (Th17) cell responses, also via expression of coinhibitory molecule TIGIT $(12,13)$. Treg cells are important for maintaining immune homeostasis and exerting immunosuppressive effects by directly recognizing tumor cells and releasing the anti-inflammatory cytokines interleukin-2 (IL-2), interleukin-10 (IL-10), transforming growth factor- $\beta$ (TGF- $\beta$ ), and interleukin-35 (IL-35) (14). Previous studies have shown that the frequency of Treg cells in tumor tissue is closely associated with the recovery of patients. In patients with HCC, an increase in the frequency of Treg cells indicates a poor prognosis (15). Furthermore, TIGIT-expressing T follicular helper cells provide support to B cell functions (16). Previous studies have shown that genetic ablation or antibody blockade of TIGIT enhances $\mathrm{CD}^{+} \mathrm{T}$ cell priming and exacerbates the severity of experimental autoimmune encephalitis and rheumatoid arthritis (17-19). In addition, the TIGIT pathway has been shown to serve an important role in regulation in other disease models, including cancer and chronic viral infection (20). However, the role of TIGIT in the pathogenesis of $\mathrm{HCC}$ remains to be elucidated.

In the present study, the expression of TIGIT and its ligand, CD155, were examined in the cancerous tissues of patients with HCC. Correlations between TIGIT $^{+} \mathrm{CD}^{+} \mathrm{T}$ cell and TIGIT $^{+}$Treg cell frequencies and clinical pathological features were also analyzed. The results showed that the TIGIT/CD155 signaling pathway may be a potential target for the diagnosis and treatment of HCC.

\section{Materials and methods}

Ethics statement. The tissues and peripheral blood of patients with HCC were collected in accordance with a protocol approved by the Ethics Committee for the Conduct of Human Research at Ningxia Medical University (Ningxia, China; no. 2015-074). Investigators obtained informed consent prior to enrolling participants in clinical trials. Patients who met the following eligibility criteria were included in the study: i) diagnosis of primary HCC identified by histopathological examination; ii) treatment with radical resection; iii) availability of complete follow-up data; iv) no pre-operative anticancer treatment, such as chemotherapy or radiotherapy; v) no history of familial malignancy or other synchronous malignancy; and vi) the patient was alive 3 months after surgery. The exclusion criteria were as follows: i) preoperative with acute infectious disease and/or blood system disease and associated complications; ii) preoperative with severe hypersplenism, kidney disease, cardiovascular and/or cerebrovascular disease, or autoimmune disease; iii) receiving adjuvant chemotherapy, such as radiotherapy and/or chemotherapy, prior to surgery; and iv) a history of steroid hormone administration in the 15 days before surgery.

HCC tissue samples and peripheral blood. A total of 77 patients with HCC (40 with liver cirrhosis and 37 with chronic hepatitis) were enrolled in the present study. Immunohistochemical analysis was performed on samples from 120 patients, including the aforementioned 77 patients, which comprised 73 cancer tissue samples and 47 adjacent tissue samples (from paracellular tissues that were $>5 \mathrm{~cm}$ from the tumor), who had undergone surgical resection at the General Hospital of Ningxia Medical University between September 2014 and January 2017. The clinical characteristics of the patients are summarized in Table I. Venous blood was obtained from 31 patients with HCC (age, 29-80 years; male: Female, 17:14) and 30 healthy controls (age, $30-55$ years; male: Female, 18:12).

Isolation of peripheral blood mononuclear cells (PBMCs). Peripheral blood samples were collected from healthy volunteers. The blood was diluted 1:1 with phosphate-buffered saline prior to the separation of PBMCs by centrifugation at $500 \mathrm{x}$ g at room temperature by Ficoll-Paque (Pharmacia, Uppsala, Sweden) density gradient centrifugation. The cells were cryopreserved in fetal bovine serum (Gibco; Thermo Fisher Scientific, Inc., Waltham MA, USA), supplemented with $10 \%$ dimethyl sulfoxide, and stored in liquid nitrogen.

Flow cytometric analysis. Isolated PBMCs were washed with PBS and centrifuged for $5 \mathrm{~min}$ at $500 \mathrm{~g}$. The supernatant was discarded and the cells were resuspended in PBS to a cell density of $1 \times 10^{6}$ cells $/ 100 \mu$ l. Cell surface staining was performed on PBMCs using the following anti-human monoclonal antibodies: CD127-AF647 (1:100; clone HIL-7R-M21, cat. no. 558598), CD4-v450 (1:500; clone RPA-T4, cat. no. 560346), CD25-APC-cy7 (1:100; clone M-A251, cat. no. 561782) and TIGIT-PE (1:300; clone 1G9, cat. no. 565168). All antibodies were purchased from BD Pharmingen ${ }^{\mathrm{TM}}$. All data were collected on a FACS Aria III ${ }^{\mathrm{TM}}$ (BD Biosciences) and were analyzed with Flow Jo software version 7.5 (Tree Star, Inc.).

Liquid chip technology. The levels of cytokines in the plasma and supernatants were determined using a cytometry bead array flex set (BD Biosciences) in accordance with the manufacturer's instructions. The assays were conducted for granulocyte-macrophage-colony-stimulating factor, interferon- $\gamma$, interleukin (IL)- $\beta$, IL-4, IL-6, IL-21, IL-10, IL-23, IL-15, IL-2IL-17A, IL-33 and tumor necrosis factor (TNF)- $\alpha$ using $50 \mu 1$ of sample and a 10-point standard curve $(0-2,500 \mathrm{pg} / \mathrm{ml})$ for each cytokine. The samples were analyzed using a flow fluorescence detector Luminex 200 (Luminex Corporation). All data were collected analyzed using Milliplex Analyst. V5.1 (Merck KGaA).

Reverse transcription-quantitative PCR (RT-qPCR) analysis. Total RNA was extracted using TRIzol total RNA isolation reagent (Takara Biotechnology Co., Ltd., Dalian, China), and reverse transcription was performed with the RT-PCR synthesis kit (Thermo Fisher Scientific, Inc.) according to the manufacturer's instructions. The cDNA was stored at $-20^{\circ} \mathrm{C}$. PCR amplification for quantification was performed with specific primers using SYBR Premix Ex Taq II (Takara Biotechnology Co., Ltd.) on the PikoReal 96 qPCR system (Thermo Fisher Scientific, Inc.) using the following thermocycling conditions: $95^{\circ} \mathrm{C}$ for $10 \mathrm{~min}$, followed by 40 cycles of $95^{\circ} \mathrm{C}$ for $30 \mathrm{sec}$, $57^{\circ} \mathrm{C}$ for $45 \mathrm{sec}$ and $72^{\circ} \mathrm{C}$ for $30 \mathrm{sec}$. The average of three independent analyses for each gene and sample was calculated 
Table I. Clinicopathologic features of patients with hepatocellular carcinoma.

Variable

Virus infection

Hepatitis B virus

Hepatitis C virus

Hepatitis $\mathrm{B}(+)$ and $\mathrm{C}$ virus

42

Liver histopathology

Chronic hepatitis

Pathological staging

Highly differentiated group

High and medium differentiation group

Medium differentiation group

Medium and low differentiation group

Low differentiation group

Size of cancer tissue (mm)

$<50$

$>50$

$\alpha$-fetoprotein $>25 \mu \mathrm{g} / \mathrm{l}$

4

another four times, and the protein bands were visualized by ECL chemiluminescence and then measured using ImageJ software 1.8.0. (National Institutes of Health, Bethesda, MD, USA). The expression levels of proteins of interest were normalized with GAPDH. Independent experiments were repeated three times for each sample.

Liver cirrhosis

and normalized to the endogenous reference control gene, $\beta$-actin. All samples were analyzed using the $2^{-\Delta \Delta C q}$ analysis method (21). The primers used for qPCR were as follows: $\beta$-actin, forward 5'-GCCAACACAGTGCTGTCTGG-3' and reverse 5'-CTCAGGAGGAGCAATGATCTTG-3'; CD4, forward 5'-AGGAAGTGAACCTGGTGGTG-3' and reverse 5'-TTGCCTCCTTGTTCTCCAGT-3'; TIGIT, forward 5'-ATG GGACGTACACTGGGAGA-3' and reverse 5'-ACTGTCGTG CAGATGACCAC-3'; CD155, forward 5'-ACTGTCACCAGC CTCTGGAT-3' and reverse 5'-GGTGAGGTTCACAGTCAG CA-3'.

Western blot analysis. The tissue total protein was extracted using RIPA buffer (Beyotime Institute of Biotechnology, Shanghai, China), and the quantity of protein was measured using an Enhanced BCA Protein Assay kit (Beyotime Institute of Biotechnology,). A total of $50 \mu \mathrm{g}$ of protein was loaded and separated on 12\% SDS-PAGE gels (Beyotime Institute of Biotechnology). The total protein was then transferred onto polyvinylidene difluoride (PVDF) membranes. The PVDF membranes were blocked using $5 \%$ skim milk powder and were then incubated with antibodies overnight at $4^{\circ} \mathrm{C}$. Rabbit CD155 monoclonal antibody (cat. no. ab103630; Abcam) and rabbit TIGIT monoclonal antibody (cat. no. ab106311; Abcam) were diluted in antibody diluent (1:500/1:1,000, respectively; Beyotime Institute of Biotechnology) and rabbit GAPDH antibody (cat. no. bs-2188R; BIOSS, Beijing, China) was diluted in antibody diluent (1:4,000). The PVDF membranes were washed four times ( 5 min each time) with TBST and then incubated with goat anti-mouse or rabbit IgG horseradish peroxidase (HRP)-conjugated secondary antibodies $(1: 4,000$; cat. nos. A21010 and A21020; Abbkine Scientific Co., Ltd.) for $1 \mathrm{~h}$ at room temperature. The PVDF membranes were washed
Immunohistochemistry. The tissues were dehydrated and embedded in paraffin wax. The sections were dewaxed in xylene and dehydrated in alcohol. Antigen retrieval was performed by incubating the slides in citric acid buffer for $10 \mathrm{~min}$. Following blocking with $3 \%$ hydrogen peroxide for $15 \mathrm{~min}$ and $5 \%$ bovine serum albumin (Beijing Solarbio Science and Technology Co., Ltd., Beijing, China), the slides were incubated overnight at $4^{\circ} \mathrm{C}$ with mouse polyclonal antibodies against CD4 (1:100; cat. no. ab133616; Abcam), CD155 (1:100; cat. no. ab103630; Abcam) and TIGIT (1:100; cat. no. ab243903; Abcam). Following rinsing with PBS, the sections were incubated with secondary antibodies from the Polink-2 plus Polymer HRP Detection System (cat. nos. ZB-2301 and ZB-2305; ZSGB-BIO) for $30 \mathrm{~min}$ at room temperature. The expression of CD4, CD155 and TIGIT was visualized by diaminobenzidine tetrahydrochloride solution (Cowin Bioscience) staining. The sections were then counterstained with hematoxylin for $5 \mathrm{~min}$. All slides were observed and images were captured under a fluorescence microscope.

Statistical analysis. All statistical calculations were performed with SPSS 23.0 software (IBM Corp.). Data are expressed as the mean \pm standard deviation (SD). Statistical differences between groups were analyzed using the Mann-Whitney U test. Spearman's rank correlation test for nonparametric data was used to analyze the relationship between the two factors. Statistical analysis was performed using GraphPad Prism software version 6.0 (GraphPad Software Inc., San Diego, CA, USA). $\mathrm{P}<0.05$ was considered to indicate a statistically significant difference.

\section{Results}

Expression of TIGIT/CD155 in cancerous tissue is significantly elevated in patients with HCC. To determine whether TIGIT/CD155 is involved in the pathogenesis of HCC, the expression of TIGIT/CD155 in paracancerous and cancerous tissues was assessed by immunohistochemistry. A previous study showed that TIGIT was expressed on $\mathrm{CD}^{+} \mathrm{T}$ cells in patients with rheumatoid arthritis (20) and on $\mathrm{CD}^{+} \mathrm{T}$ cells in both healthy individuals and in patients with melanoma $(22,23)$. In order to further understand the aggregation of inflammation in tumor sites, the expression of $\mathrm{CD}^{+} \mathrm{T}$ cells was also examined in patients with HCC. As shown in Fig. 1A and B, the expression of CD4 was distinctly elevated in the liver tumor tissues compared with that in the paracancerous tissues. In the cancerous tissue, as the degree of malignancy increased, the number of $\mathrm{CD}^{+}$cells gradually increased, indicating that the interaction between the tumor cells and effector cells determines disease prognosis. The expression levels of TIGIT and CD155 were increased in liver cancer tissues. Of note, the expression of TIGIT gradually increased in liver cancer tissues as the degree of tumor cell differentiation changed from high 
A

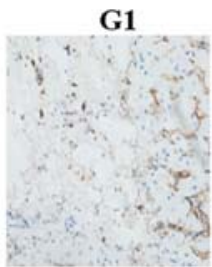

G4

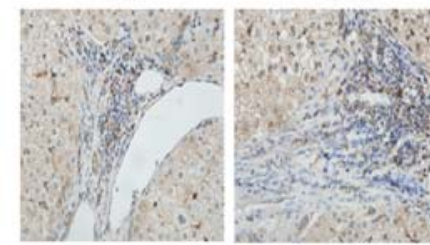

C

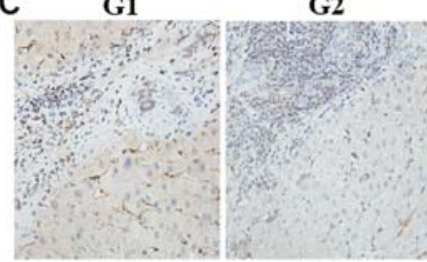

G4

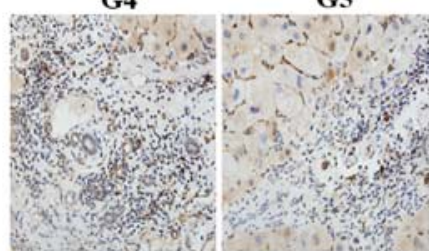

$\mathrm{E}$

E

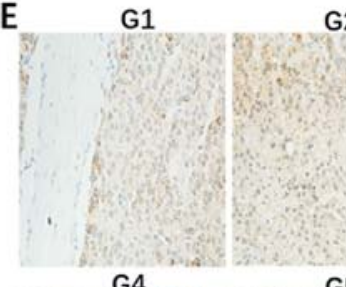

G4

G5

G2

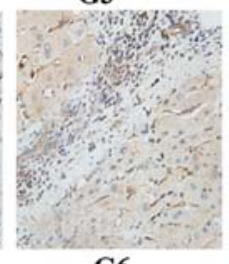

G6

G3

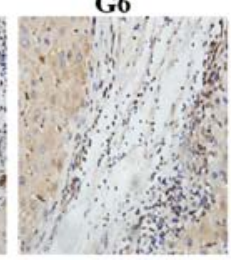

G6
G3

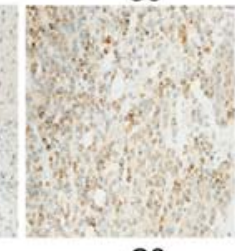

B
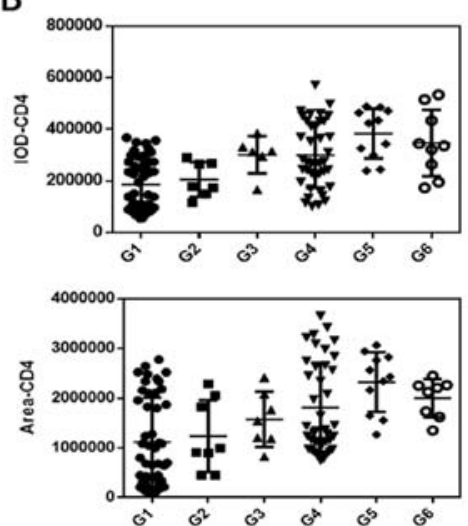

D
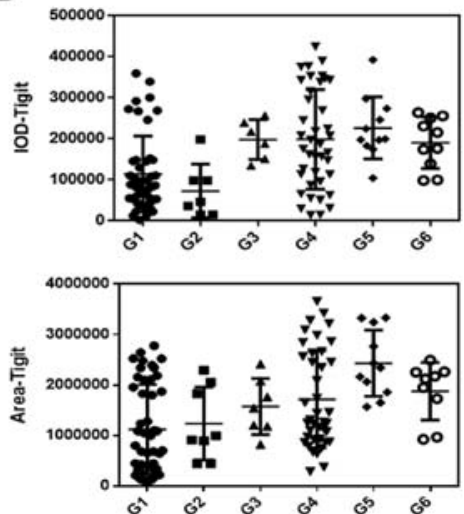

$\mathrm{F}$
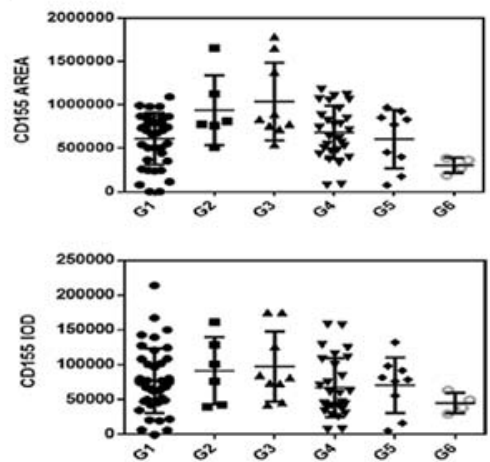

G

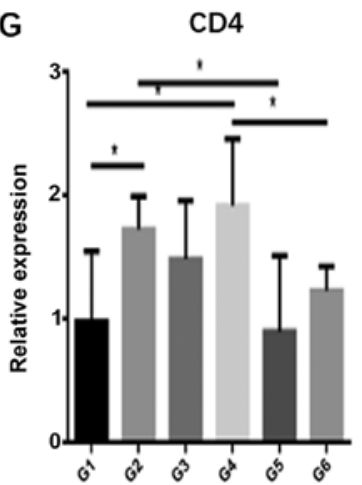

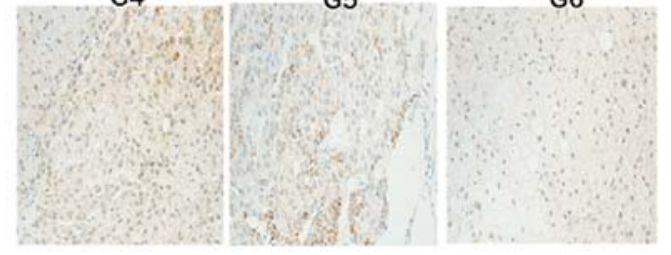
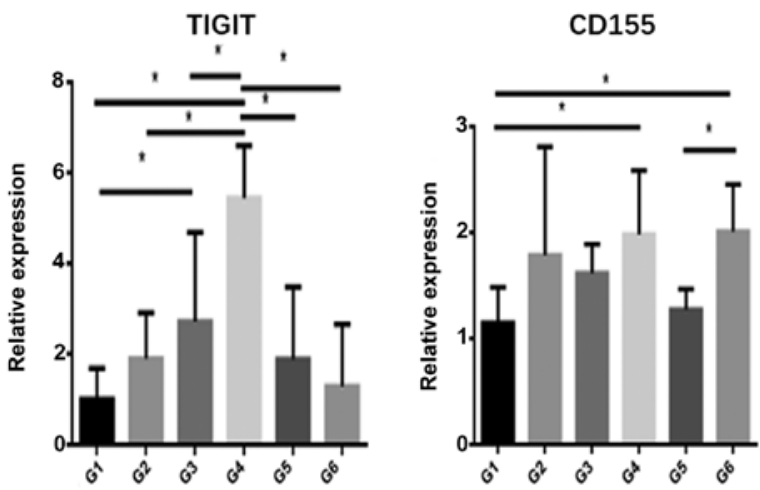

Figure 1. Expression of CD4, TIGIT and CD155 in HCC tissues at different pathological stages and adjacent tissues. Paracancerous tissues (G1) and HCC tissues (G2-6) were stained by immunohistochemistry (x400 magnification). (A) Staining of CD4 and (B) statistical analysis of CD4-positive dots in paracancerous and HCC tissues. (C) Staining of TIGIT and (D) statistical analysis of TIGIT-positive dots in paracancerous and HCC tissues. (E) Staining of CD155 and (F) statistical analysis of CD155-positive dots in paracancerous and HCC tissues. (G) Relative mRNA expression levels of CD4, TIGIT and CD155. All data from at least three independent experiments were measured by the band density, which were normalized to GAPDH. Bar graphs (mean \pm SEM) and representative images are shown. "P<0.05. G1, paracancerous tissue; G2, highly differentiated group; G3, high and medium differentiation; G4, medium differentiation; G5, medium and low differentiation; G6, low differentiation. HCC, hepatocellular carcinoma; TIGIT, T cell immunoglobulin and ITIM domain. 

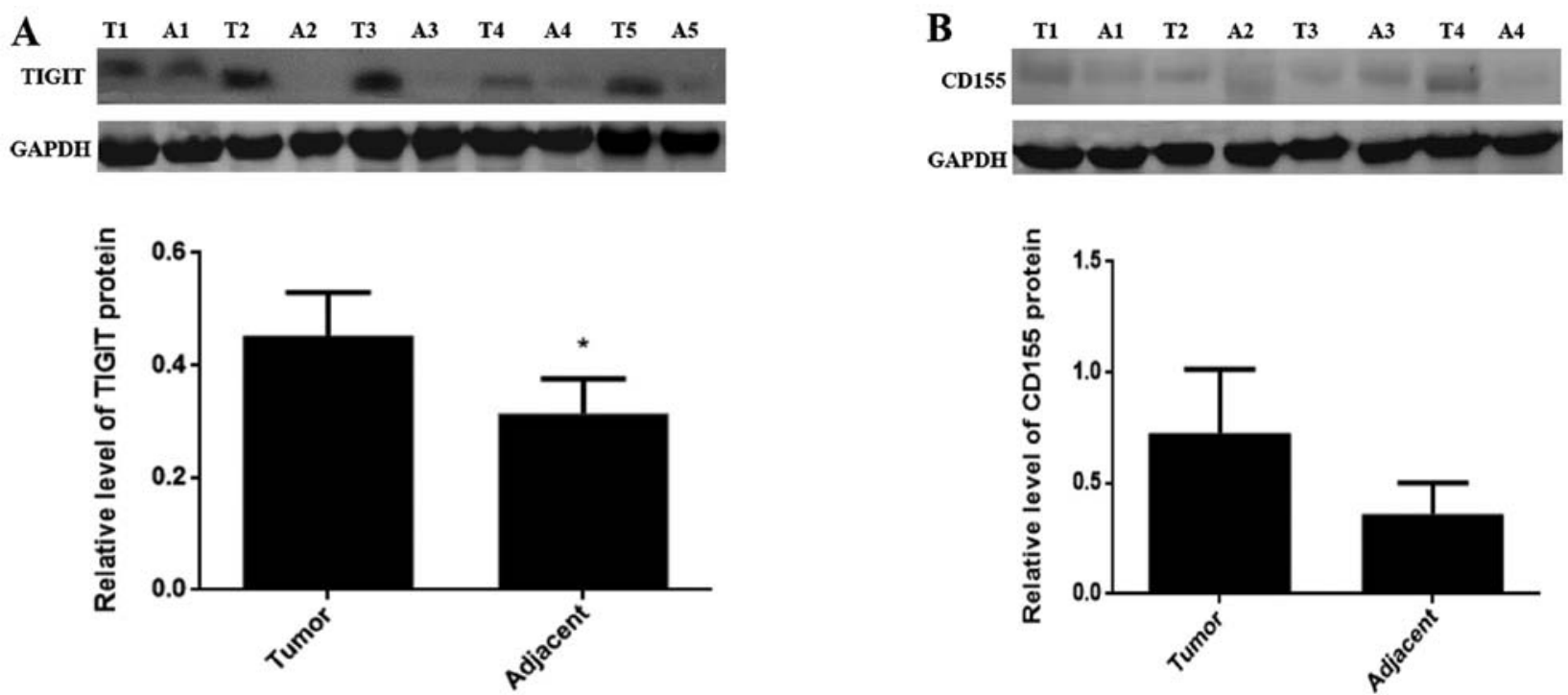

Figure 2. Expression of TIGIT and CD155 in different patients with HCC. (A) Protein expression of TIGIT was detected by western blotting. (B) Protein expression of CD155 was detected by western blotting. Data are expressed as the mean $\pm \mathrm{SD}$ (" $\mathrm{P}<0.05$ vs. tumor). 1, 2, 3, 4 and 5 refer to patients $1,2,3,4$ and 5 , respectively. T, tumor tissue; A, adjacent tissue; TIGIT, T cell immunoglobulin and ITIM domain.

to low, although the expression of CD155 was highest in the high-medium differentiation stage; generally, the expression of CD155 gradually decreased as differentiation increased (Fig. 1C-F). The molecular mRNA expression levels of TIGIT and CD155 were detected by RT-qPCR analysis. The results showed that the mRNA expression levels of CD4, TIGIT and CD155 were higher in cancerous tissues than those in the paracancerous tissues (Fig. 1G). This suggests that the TIGIT/CD155 pathway may be involved in the pathogenesis of HCC.

Expression of TIGIT and CD155 in different patients with $H C C$. In order to confirm the roles of TIGIT and CD155 in HCC, the protein expression levels of TIGIT and CD155 were examined via western blotting in cancerous and adjacent tissues from different patients. As shown in Fig. 2A and B, the expression levels of TIGIT and CD155 in the cancerous tissues were higher than those in the paracancerous tissues.

TIGIT $^{+} \mathrm{CD}^{+}$T cell and TIGIT ${ }^{+}$Treg cell frequency shift in patients with HCC. It is well known that TIGIT is expressed on activated $\mathrm{T}$ cells, including $\mathrm{CD} 4^{+} \mathrm{T}$ and Treg cells. In the present study, the frequency of TIGIT $^{+} \mathrm{CD}^{+} \mathrm{T}$ and $\mathrm{TIGIT}^{+}$Treg cells was altered in patients with HCC. PBMCs were extracted from the peripheral blood 5 days before surgery and 5 days after surgery for flow cytometry. The gated strategy of flow cytometry is shown in Fig. 3A. The frequency of CD4 cells in patients with $\mathrm{HCC}$ was higher than that in the normal control group and decreased following surgery (Fig. 3B). The frequency of $\mathrm{TIGIT}^{+} \mathrm{CD}^{+} \mathrm{T}$ cells was increased in patients with $\mathrm{HCC}$ following surgery (Fig. 3B). Another important finding was that the frequency of TIGIT $^{+}$Treg cells was higher in patients with $\mathrm{HCC}$ than that in the normal control group and was decreased following surgery (Fig. 3B). The secretion of cytokines from different $\mathrm{T}$ cell subpopulations was then analyzed. The results showed that the expression of the pro-inflammatory cytokine IL-17a was increased ( $\mathrm{P}=0.047$; Fig. 3C), the expression of
IL-6 was increased ( $\mathrm{P}=0.043$; Fig. 3C), and the expression of the anti-inflammatory factor IL-10 was decreased $(\mathrm{P}=0.034$; Fig. 3D) following surgery. These results indicate that the frequency and functional changes of $\mathrm{TIGIT}^{+} \mathrm{CD} 4^{+} \mathrm{T}$ cells and TIGIT $^{+}$Treg cells are involved in the pathogenesis of HCC. In addition, when the balance between subsets of $\mathrm{T}$ cells was disrupted, the functional changes of $\mathrm{T}$ cell subsets mediated the regulation of tumor development.

Correlation between $\mathrm{TIGIT}^{+} \mathrm{CD}^{+} \mathrm{T}$ cells and $\mathrm{TIGIT}^{+}$Treg cell frequency and AFP. AFP is the most common clinical index used in the diagnosis of liver cancer. Therefore, a possible correlation between the expression of TIGIT $^{+} \mathrm{CD}^{+}$ $\mathrm{T}$ and TIGIT $^{+}$Treg cells and the clinical indicator, AFP, was examined. The results showed that the frequency of TIGIT $^{+}$ $\mathrm{CD}^{+}{ }^{+} \mathrm{T}$ cells (Fig. 4A) and TIGIT ${ }^{+}$Treg cells (Fig. 4B) in the peripheral blood correlated positively with AFP, which may contribute to an early diagnosis of HCC.

\section{Discussion}

The incidence of HCC has been increasing, which is a concern due to HCC being a disease with a high mortality rate (24). Liver cancer is one example of an inflammation-related type of cancer and $>90 \%$ of cases of liver cancer are caused by liver damage and infection (25), which contributes to the formation, progression, escape, proliferation, invasion, angiogenesis and metastasis of tumor cells. Among inflammatory aspects, the activation of nuclear transcription factor (NF)- $\mathrm{B}$ contributes to inflammation-induced tumors. In addition, tumor suppressor genes with specific mutations or abnormal expression can activate $\mathrm{NF}-\kappa \mathrm{B}$ and increase the possibility of immune evasion, which can promote the development of HCC (26). Therefore, the liver remains in an inflammatory environment for a long period of time, causing necrosis that develops into cirrhosis and eventually leads to liver cancer (27). At present, there is no optimal diagnostic test or therapy for liver cancer. Therefore, 

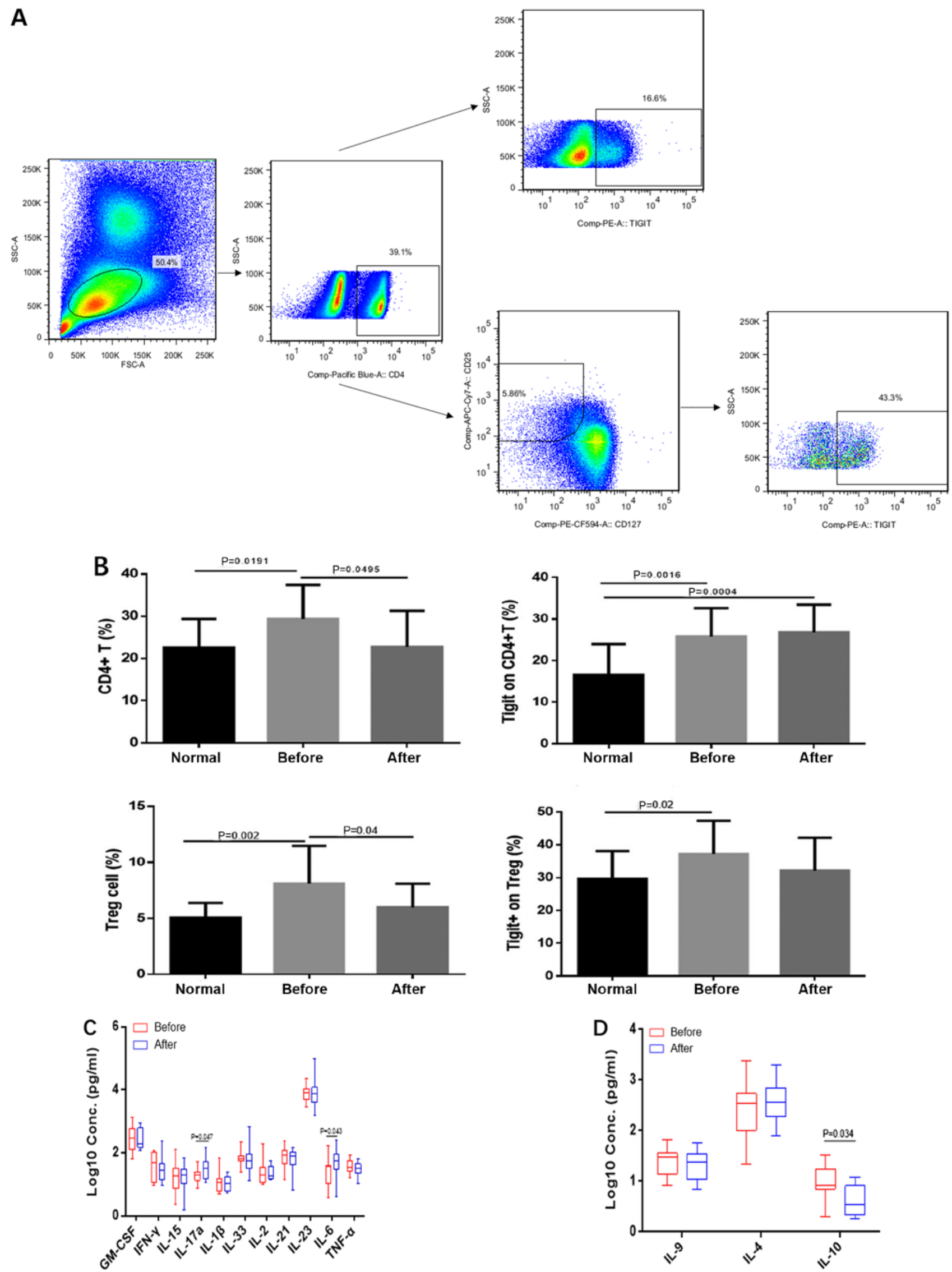

Figure 3. Changes in TIGIT $^{+} \mathrm{CD}^{+} \mathrm{T}$ cell and TIGIT $^{+}$Treg cell numbers. TIGIT ${ }^{+} \mathrm{CD}^{+} \mathrm{T}$ cells and $\mathrm{TIGIT}^{+}$Treg cells from normal control $(\mathrm{n}=30)$ and HCC $(n=31)$ groups were examined by flow cytometry. (A) Images shows the gating strategy for peripheral blood mononuclear cells by flow cytometry. (B) Frequencies of $\mathrm{CD}^{+}{ }^{+}$cells, TIGIT $^{+} \mathrm{CD}^{+}{ }^{+} \mathrm{T}$ cells, Treg cells and TIGIT $^{+}$Treg cells in HCC peripheral blood (pre- and post-surgery) and healthy volunteers. (C) Changes in pro-inflammatory cytokine concentrations pre-and post-surgery. (D) Changes in anti-inflammatory cytokine concentrations pre- and post-surgery. The data indicate the mean \pm SD. HCC, hepatocellular carcinoma; Treg cell, regulatory T cell; TIGIT, T cell immunoglobulin and ITIM domain; granulocyte-macrophage colony-stimulating factor; IFN, interferon; IL, interleukin; TNF, tumor necrosis factor. 

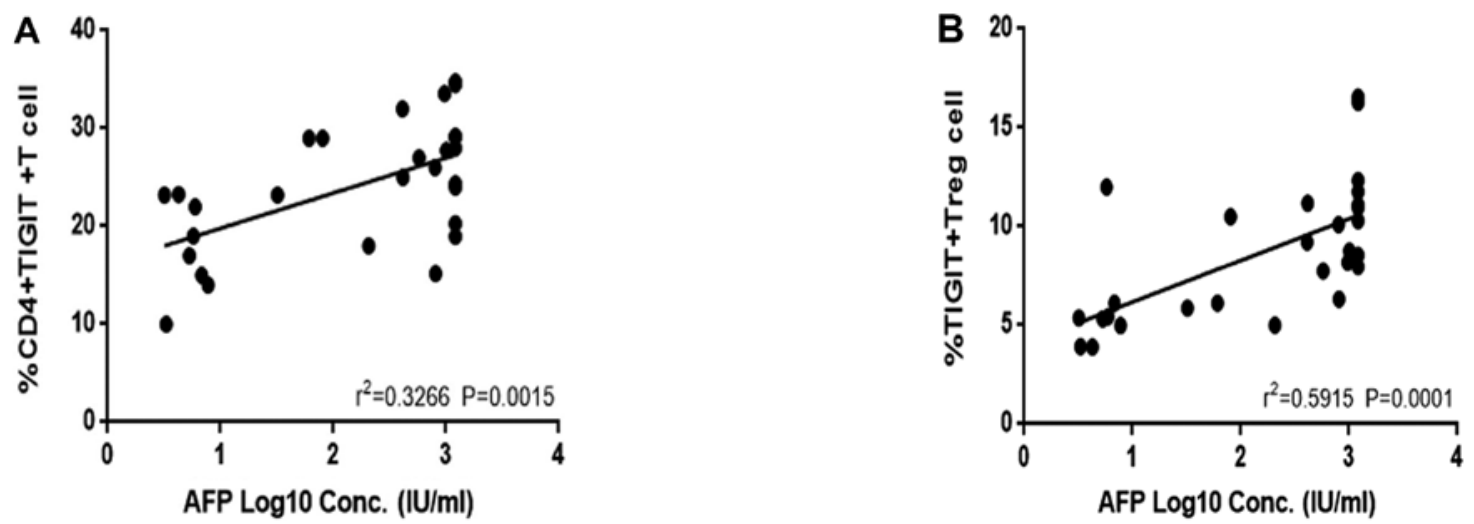

Figure 4. Correlation between TIGIT $^{+} \mathrm{CD}^{+} \mathrm{T}$ and $\mathrm{TIGIT}^{+}$Treg cell frequency and AFP. (A) Correlation between the expression of TIGIT on CD4 ${ }^{+} \mathrm{T}$ cells and AFP from patients with HCC. (B) Correlation between the frequency of TIGIT ${ }^{+}$Treg cells in peripheral blood mononuclear cells and AFP from patients with HCC. TIGIT, T cell immunoglobulin and ITIM domain; AFP, $\alpha$-fetoprotein; HCC, hepatocellular carcinoma.

identifying novel methods to diagnose and treat liver cancer is vital. The co-inhibitory receptor pathway has attracted widespread attention as a potential target of immune regulation. Significant success has been achieved with the use of co-inhibitory receptor pathways, such as cytotoxic T lymphocyte antigen 4 (CTLA-4) and programmed death-1 (PD-1), in the treatment of cancer (28-30). TIGIT is a recently identified co-inhibitory receptor that is expressed on activated $\mathrm{T}$ cells, Treg cells, NK cells and tumor cells $(8,11,19,31)$. Similar to CTLA-4 and CD28, TIGIT competes with its costimulatory counterpart, CD226, for the same ligands (CD155 and CD112) and mediates immune suppression in tumors and chronic infections (32). In the present study, as the degree of malignancy increased in cancerous tissues, the number of $\mathrm{CD}^{+}$cells gradually increased, indicating that the interaction between tumor cells and immune cells determines the prognosis of HCC. Previous studies have shown that HCC can evade host immune surveillance and has the ability of self-protection $(33,34)$. In addition, HCC cells avoid immune attack through the secretion of immunosuppressive cytokines, abnormal expression of antigens and changes to the local immune microenvironment (34). Evidence has also demonstrated that immunosuppressive factors expressed by tumor cells, which inhibit APC or T cell function, suppress antigen presentation and immune response and facilitate the immune evasion of tumor cells $(35,36)$. A number of studies have also found that the overexpression of TIGIT reduces the function of $\mathrm{CD} 4^{+} \mathrm{T}$ cells in rheumatoid arthritis (20). The expression of TIGIT on $\mathrm{CD} 4^{+} \mathrm{T}$ cells was found to be significantly increased in patients with systemic lupus erythematosus and correlated with the activity of the disease (37). CD155/TIGIT signaling has also been found to regulate $\mathrm{CD} 8^{+} \mathrm{T}$ cell metabolism and promote tumor progression in human gastric cancer (8). A noteworthy finding in the present study was that TIGIT and its ligand CD155 were upregulated in cancerous tissues from patients with HCC as the degree of cancerous differentiation reduced, suggesting that the TIGIT/CD155 pathway is involved in the pathogenesis of HCC. Other co-inhibitory receptors have also been reported, such as PD-1 marking dysfunctional regulatory $\mathrm{T}$ cells in malignant gliomas (38). These results suggest that the TIGIT/CD155 pathway serves an important role in HCC.
As is well known, the tumor immune microenvironment is produced by the interaction among tumor cells, immune cells and the tumor stroma. In addition to effector T cells, the existence of Treg cells, which negatively regulate immune responses, inhibits effector T cells. The expression of TIGIT on $\mathrm{CD} 4^{+} \mathrm{T}$ and Treg cells was detected by flow cytometry in peripheral blood from patients with HCC prior to and following surgery. The frequency of $\mathrm{TIGIT}^{+} \mathrm{CD}^{+} \mathrm{T}$ cells increased prior to surgery, and the frequency of $\mathrm{TIGIT}^{+}$Treg cells decreased following surgery, compared with the frequency in healthy controls. This indicates that these cells may be involved in tumor immune evasion and that the expression of TIGIT affects the functions of $\mathrm{CD}^{+}$effector cells and Treg cells, which mediates HCC immune evasion. The immune environment in the body is disordered, and the balance of cytokines secreted by immune effector cells alters with the development of HCC tumors. In the present study, the levels of IL-17a and IL-6 in patients with HCC increased following surgery, indicating that antitumor immunity was reversed following surgery and that immune defense in the body was restored. IL-10 is one of the immunosuppressive cytokines secreted by Treg cells to suppress the immune response. The decreased level of IL-10 in patients postoperatively suggests that the immune response had recovered.

AFP is the most common tumor marker for the diagnosis of primary liver cancer. The positive rate and specificity of AFP in liver cancer is $\sim 70 \%$. In the present study, TIGIT $^{+}$ $\mathrm{CD}^{+}{ }^{+} \mathrm{T}$ and $\mathrm{TIGIT}^{+}$Treg were positively correlated with AFP, suggesting that TIGIT $^{+} \mathrm{T}$ cells are of potential clinical value in the diagnosis of HCC. Further investigations based on medical evidence are required to examine this application.

Taken together, the human immune system is disordered in liver cancer. The number and function of immune cells are altered due to the high expression of TIGIT, particularly on effector $\mathrm{T}$ cells and Treg cells, which serve important roles in the tumor microenvironment. The interaction between TIGIT $^{+}$tumor cells and TIGIT $^{+} \mathrm{T}$ cells appears to determine the severity and prognosis of HCC. In addition, the expression of TIGIT on $\mathrm{CD}^{+} \mathrm{T}$ and Treg cells was positively correlated with AFP and may be a potential indicator for HCC detection. Therefore, the expression of TIGIT may serve as a key molecule for the diagnosis and treatment of HCC in the future. 


\section{Acknowledgements}

The authors would like to thank Richard Ho Lye Yin (Ph.D) and Ms Margaret Vera Trelfa from Ningxia Medical University for making corrections to the text.

\section{Funding}

The present study received financial support from the National Natural Science Foundation of China (grant nos. 81560466,81760437,31860695 and 81560504), the Natural Science Foundation Project of Ningxia (grant no. NZ16055), the Innovation and Entrepreneurship for Returned Students Project of Ningxia, China (2017) (grant no. YXW2017084) and the Construction of First-class Disciplines in Ningxia University (Basic Medicine in Western China) grant no. NXYLXK2017B07).

\section{Availability of data and materials}

The datasets used and/or analyzed during the current study are available from the corresponding author on reasonable request.

\section{Authors' contributions}

XD and CS contributed to study design, drafted the manuscript and gave final approval of the manuscript; JL, JC and QZ contributed to study design and data analysis, and gave final approval of the manuscript; $\mathrm{XY}$ and BM contributed to experimental operation and gave final approval of the manuscript; $\mathrm{ZL}$ and YD acquired the data and revised the manuscript for important intellectual content. All authors gave final approval of the version to be published and agreed to be accountable for all aspects of the work.

\section{Ethics approval and consent to participate}

The tissues and peripheral blood of patients with HCC were collected in accordance with a protocol approved by the Ethics Committee for the Conduct of Human Research at Ningxia Medical University (Ningxia, China; no. 2015-074). Investigators obtained informed consent before enrolling participants in clinical trials.

\section{Patient consent for publication}

Not applicable.

\section{Competing interests}

The authors declare that they have no competing interests.

\section{References}

1. Ferlay J, Soerjomataram I, Dikshit R, Eser S, Mathers C, Rebelo M, Parkin DM, Forman D and Bray F: Cancer incidence and mortality worldwide: Sources, methods and major patterns in GLOBOCAN 2012. Int J Cancer 136: E359-E386, 2015.

2. Mazzanti R, Gramantieri L and Bolondi L: Hepatocellular carcinoma: Epidemiology and clinical aspects. Mol Aspects Med 29: $130-143,2008$
3. El-Serag HB: Epidemiology of hepatocellular carcinoma in USA. Hepatol Res 37 (Suppl 2): S88-S94, 2007.

4. El-Serag HB and Rudolph KL: Hepatocellular carcinoma: Epidemiology and molecular carcinogenesis. Gastroenterology 132: 2557-2576, 2007.

5. Greten TF, Duffy AG and Korangy F: Hepatocellular Carcinoma from an immunologic perspective. Clin Cancer Res 19: 6678-6685, 2013.

6. Parkin DM, Bray F, Ferlay $\mathrm{J}$ and Pisani P: Estimating the world cancer burden: Globocan 2000. Int J Cancer 94: 153-156, 2001.

7. Boles KS, Vermi W, Facchetti F, Fuchs A, Wilson TJ, Diacovo TG, Cella $\mathrm{M}$ and Colonna $\mathrm{M}$ : A novel molecular interaction for the adhesion of follicular CD4 T cells to follicular DC. Eur J Immunol 39: 695-703, 2009.

8. He W, Zhang H, Han F, Chen X, Lin R, Wang W, Qiu H, Zhuang Z, Liao Q, Zhang W, et al: CD155T/TIGIT signaling regulates CD8(+) T-cell metabolism and promotes tumor progression in human gastric cancer. Cancer Res 77: 6375-6388, 2017.

9. Joller N, Hafler JP, Brynedal B, Kassam N, Spoerl S, Levin SD Sharpe AH and Kuchroo VK: Cutting Edge: TIGIT Has T cell-intrinsic inhibitory functions. J Immunol 186: 1338-1342, 2011.

10. Yu X, Harden K, Gonzalez LC, Francesco M, Chiang E, Irving B, Tom I, Ivelja S, Refino CJ, Clark $\mathrm{H}$, et al: The surface protein TIGIT suppresses $\mathrm{T}$ cell activation by promoting the generation of mature immunoregulatory dendritic cells. Nat Immunol 10: 48-57, 2008.

11. Levin SD, Taft DW, Brandt CS, Bucher C, Howard ED, Chadwick EM, Johnston J, Hammond A, Bontadelli K, Ardourel D, et al: Vstm3 is a member of the CD28 family and an important modulator of T-cell function. Eur J Immunol 41: 902-915, 2011.

12. Joller N, Lozano E, Burkett PR, Patel B, Xiao S, Zhu C, Xia J, Tan TG, Sefik E, Yajnik V, et al: Treg cells expressing the coinhibitory molecule TIGIT selectively inhibit proinflammatory Th1 and Th17 cell responses. Immunity 40: 569-581, 2014.

13. Kurtulus S, Sakuishi K, Ngiow SF, Joller N, Tan DJ, Teng MW, Smyth MJ, Kuchroo VK and Anderson AC: TIGIT predominantly regulates the immune response via regulatory $\mathrm{T}$ cells. J Clin Invest 125: 4053-4062, 2015.

14. Trehanpati $\mathrm{N}$ and Vyas $\mathrm{AK}$ : Immune regulation by $\mathrm{T}$ regulatory cells in hepatitis B virus-related inflammation and cancer. Scand J Immunol 85: 175-181, 2017.

15. Kurose K, Ohue Y, Sato E, Yamauchi A, Eikawa S, Isobe M, Nishio Y, Uenaka A, Oka M and Nakayama E: Increase in activated treg in til in lung cancer and in vitro depletion of treg by ADCC using an antihuman CCR4 mAb (KM2760). J Thorac Oncol 10: 74-83, 2015.

16. Godefroy E, Zhong H,Pham P, Friedman D and Yazdanbakhsh K: TIGIT-positive circulating follicular helper $\mathrm{T}$ cells display robust B-cell help functions: Potential role in sickle cell alloimmunization. Haematologica 100: 1415-1425, 2015.

17. Goding SR, Wilson KA, Xie Y, Harris KM, Baxi A, Akpinarli A, Fulton A, Tamada K, Strome SE and Antony PA: Restoring immune function of tumor-specific $\mathrm{CD}^{+} \mathrm{T}$ cells during recurrence of melanoma. J Immunol 190: 4899-4909, 2013.

18. Stengel KF, Harden-Bowles K, Yu X, Rouge L, Yin J, Comps-Agrar L, Wiesmann C, Bazan JF, Eaton DL and Grogan JL: Structure of TIGIT immunoreceptor bound to poliovirus receptor reveals a cell-cell adhesion and signaling mechanism that requires cis-trans receptor clustering. Proc Natl Acad Sci USA 109: 5399-5404, 2012.

19. Stanietsky N, Simic H, Arapovic J, Toporik A, Levy O, Novik A, Levine Z, Beiman M, Dassa L, Achdout H, et al: The interaction of TIGIT with PVR and PVRL2 inhibits human NK cell cytotoxicity. Proc Natl Acad Sci USA 106: 17858-17863, 2009.

20. Zhao W, Dong Y, Wu C, Ma Y, Jin Y and Ji Y: TIGIT overexpression diminishes the function of $\mathrm{CD} 4 \mathrm{~T}$ cells and ameliorates the severity of rheumatoid arthritis in mouse models. Exp Cell Res 340: 132-138, 2016.

21. Livak KJ and Schmittgen TD: Analysis of relative gene expression data using real-time quantitative PCR and the 2(-Delta Delta C(T)) method. Methods 25: 402-408, 2001.

22. Chauvin JM, Pagliano O, Fourcade J, Sun Z, Wang H, Sander C, Kirkwood JM, Chen TH, Maurer M, Korman AJ and Zarour HM: TIGIT and PD-1 impair tumor antigen-specific CD8(+) T cells in melanoma patients. J Clin Invest 125: 2046-2058, 2015.

23. Song Y, Wang B, Song R, Hao Y, Wang D, Li Y, Jiang Y, Xu L, Ma Y, Zheng H, et al: T-cell Immunoglobulin and ITIM Domain Contributes to CD8(+) T-cell Immunosenescence. Aging Cell 17, 2018. 
24. Imbeaud S, Ladeiro Y and Zucman-Rossi J: Identification of novel oncogenes and tumor suppressors in hepatocellular carcinoma. Semin Liver Dis 30: 75-86, 2010.

25. Matsuzaki K, Murata M, Yoshida K, Sekimoto G, Uemura Y, Sakaida N, Kaibori M, Kamiyama Y, Nishizawa M, Fujisawa J, et al: Chronic inflammation associated with hepatitis $\mathrm{C}$ virus infection perturbs hepatic transforming growth factor beta signaling, promoting cirrhosis and hepatocellular carcinoma. Hepatology 46: 48-57, 2007.

26. Yang L and Karin M: Roles of tumor suppressors in regulating tumor-associated inflammation. Cell Death Differ 21: 1677-1686, 2014.

27. Sanz-Cameno P, Trapero-Marugán M, Chaparro M, Jones EA and Moreno-Otero R: Angiogenesis: From chronic liver inflammation to hepatocellular carcinoma. J Oncol 2010: 272170, 2010

28. Pedroza-Gonzalez A, Zhou G, Vargas-Mendez E, Boor PP, Mancham S, Verhoef C, Polak WG, Grünhagen D, Pan Q, Janssen $\mathrm{H}$, et al: Tumor-infiltrating plasmacytoid dendritic cells promote immunosuppression by $\mathrm{Trl}$ cells in human liver tumors. Oncoimmunology 4: e1008355, 2015.

29. Callahan MK, Postow MA and Wolchok JD: CTLA-4 and PD-1 pathway blockade: Combinations in the clinic. Front Oncol 4: $385,2014$.

30. Hamid O, Robert C, Daud A, Hodi FS, Hwu WJ, Kefford R Wolchok JD, Hersey P, Joseph RW, Weber JS, et al: Safety and tumor responses with lambrolizumab (anti-PD-1) in melanoma. N Engl J Med 369: 134-144, 2013.

31. Stanietsky N, Rovis TL, Glasner A, Seidel E, Tsukerman P, Yamin R, Enk J, Jonjic S and Mandelboim O: Mouse TIGIT inhibits NK-cell cytotoxicity upon interaction with PVR. Eur J Immunol 43: 2138-2150, 2013.
32. Johnston RJ, Comps-Agrar L, Hackney J, Yu X, Huseni M, Yang Y, Park S, Javinal V, Chiu H, Irving B, et al: The immunoreceptor TIGIT regulates antitumor and antiviral $\mathrm{CD}^{+} \mathrm{T}$ cell effector function. Cancer Cell 26: 923-937, 2014.

33. Jenne $C N$ and Kubes P: Immune surveillance by the liver. Nat Immunol 14: 996-1006, 2013

34. Nemeth E, Baird AW and O'Farrelly C: Microanatomy of the liver immune system. Semin Immunopathol 31: 333-343, 2009.

35. Zhong Z, Carroll KD, Policarpio D, Osborn C, Gregory M, Bassi R, Jimenez X, Prewett M, Liebisch G, Persaud K, et al: Anti-transforming growth factor beta receptor II antibody has therapeutic efficacy against primary tumor growth and metastasis through multieffects on cancer, stroma, and immune cells. Clin Cancer Res 16: 1191-1205, 2010.

36. Zakrzewski PK, Cygankiewicz AI, Mokrosiński J, NowackaZawisza M, Semczuk A, Rechberger T and Krajewska WM: Expression of endoglin in primary endometrial cancer. Oncology 81: 243-250, 2011.

37. Mao L, Hou H, Wu S, Zhou Y, Wang J, Yu J, Wu X, Lu Y, Mao L, Bosco MJ, et al: TIGIT signalling pathway negatively regulates $\mathrm{CD}^{+} \mathrm{T}$-cell responses in systemic lupus erythematosus. Immunology 151: 280-290, 2017.

38. Lowther DE, Goods BA, Lucca LE, Lerner BA, Raddassi K, van Dijk D, Hernandez AL, Duan X, Gunel M, Coric V, et al: PD-1 marks dysfunctional regulatory T cells in malignant gliomas. JCI Insight 1: pii: e85935, 2016.

This work is licensed under a Creative Commons Attribution-NonCommercial-NoDerivatives 4.0 International (CC BY-NC-ND 4.0) License. 